\title{
A DINÂMICA DO USO E COBERTURA DO SOLO NO LAGO POOPÓ (BOLÍVIA) ENTRE 1985 E 2017 UTILIZANDO CLASSIFICAÇÃO VOLTADA AO OBJETO EM DADOS LANDSAT
}

\author{
DYNAMICS OF LAND USE AND COVERAGE IN LAKE POOPÓ (BOLIVIA) BETWEEN 1985 \\ AND 2017 USING OBJECT-ORIENTED CLASSIFICATION IN LANDSAT DATA
}

\author{
Florença das Graças MOURA ${ }^{1}$, Álvaro Xavier FERREIRA ${ }^{1}$, Tati ALMEIDA ${ }^{1}$, Jérémie \\ GARNIER $^{1}$, Rejane Ennes CICERELLI ${ }^{1}$, Frédéric SATGÉ ${ }^{1}$, Marie Paule BONNET ${ }^{2}$ \\ ${ }^{1}$ Universidade de Brasília, Instituto de Geociências, Campus Universitário Darcy Ribeiro, Instituto Central de Ciências - Ala Central, \\ Asa Norte. Brasília - DF. E-mails: fmouraunb@gmail.com; alvaroxavier.f@gmail.com; garnier@unb.br; rejaneig@unb.br; \\ frederic.satge@gmail.com \\ ${ }^{2}$ Institut de Recherche pour le Développement, UMR 228 Espace-DEV. 500 Rue J.F. Bretoni, Maison de la Télédétection, \\ Montpellier, France. E-mail: marie-paule.bonnet@ ird.fr
}

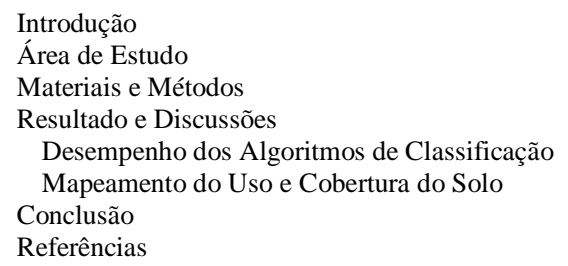

\begin{abstract}
RESUMO - O lago Poopó é o segundo maior lago da Bolívia e atualmente vem passando por uma forte crise hídrica que alguns autores associam diretamente a mudança de ocupação da terra. Neste trabalho foi realizada a classificação do uso e cobertura do solo na subbacia P6 do lago entre os anos de 1985 e 2017. Foi analisado o desempenho dos classificadores SVM (Support Vector Machines), KNN (K-Nearest Neighbor) e MaxVer (Máxima Verossimilhança). A classificação que obteve melhor acurácia foi a gerada pelo classificador SVM, em que o valor do índice Kappa foi de 82,28\% e 83,7\% para as imagens Landat-5 e Landsat-8, respectivamente, e a exatidão global foi de $92 \%$ para ambas as imagens. A partir das classificações geradas foi verificado que as maiores alterações se deram nas classes de vegetação nativa, agricultura e área úmida. A perda de área úmida na sub-bacia vem ocorrendo desde 1995,15 anos antes do aumento da atividade agrícola, que começou a partir de 2010. Assim, diversos são os fatores que podem estar contribuindo com essa redução acelerada dos corpos de água, como variações climáticas locais e as atividades antrópicas que interferem no ciclo hidrológico de forma regional.
\end{abstract}

Palavras-Chave: Lago Poópo, Classificação do uso e cobertura do solo, Envi FX, Classificadores Automáticos, Classificação Orientada a Objetos.

\begin{abstract}
The Poopó Lake is the second largest lake in Bolivia and is currently undergoing a severe water crisis, that some authors directly associate the change of land occupation. In this work a classification of land use and coverage was carried out in the P6 subbasin of the lake between the years of 1985 and 2017. Initially, the performance of the algorithms was analyzed: SVM (Support Vector Machines), KNN (K-Nearest Neighbor) e MaxVer (Maximum-Likelihood). The classification with better acuracy was performed by the SVM algorithm, where the Kappa index value was $82.28 \%$ and $83.7 \%$ for Landat-5 and Landsat-8 images respectively, and Global Accuracy was $92 \%$ for both images. With the classification it was verified that the most significant changes occurred in the native vegetation, agriculture and wetland areas. The loss of wetland area in the sub-basin has been occurring since 1995, 15 years before the increase in agricultural activity, which started of 2010. Thus, several factors may be contributing to this accelerated reduction in the lake, for example, the local climatic variations and the anthropic activities that interfere in the hydrological cycle regionally.

Keywords: Poopó Lake, Classification of land use and coverage, Envi FX, Automatic Classifiers, Object-Oriented Classification.
\end{abstract}

\section{INTRODUÇÃO}

O uso e ocupação inadequados do solo podem comprometer a integridade das bacias hidrográficas (Restrepo et al., 2017, Turner et al., 2007, Donatio et al., 2005). A agricultura extensiva e a remoção parcial ou total da vegetação nativa, por exemplo, pode acelerar o processo natural de lixiviação e erosão do solo que leva ao assoreamento dos corpos d'água, além de aumentar a disponibilidade de água a processos de evapotranspiração (Blaikie e Brookfield, 2015; Bommarco et al., 2013). A compreensão da dinâmica do uso da terra é de fundamental importância para a gestão dos recursos naturais, pois a falta de planejamento assim como o mau uso do solo, pode alterar todo o ciclo hidrológico de uma área (Small et al., 2001).

A utilização de imagens de sensores remotos 
na obtenção das classificações de uso e cobertura do solo para o monitoramento de recursos naturais tem resultados consolidados na literatura (Nunes \& Roig, 2014; Blaschke \& Kux, 2007). Os avanços tecnológicos na área de sensoriamento remoto nos últimos anos têm possibilitado a aquisição cada vez mais precisa e acurada das informações de imagens de satélite. Os sensores atuais possuem melhores resoluções espaciais, espectrais, radiométricas e temporais, permitindo a extração fidedigna de informação (Sano et al., 2009).

As metodologias para a extração de informações utilizando imagens devem ser sistematicamente testadas para replicação em diferentes sensores e áreas geográficas (Meneses \& Almeida, 2012). A utilização de técnicas de classificação de imagens voltada ao objeto vem mostrando resultados promissores no mapeamento do uso da terra com imagens de média resolução espacial e espectral (Luz et al., 2010; Fernandes et al., 2012). A vantagem dessa técnica de interpretação automática de imagens é o aumento da produtividade e precisão do trabalho, bem como na redução da subjetividade do resultado (Camargo et al., 2012).

Nesse sentido, esse trabalho pretende utilizar técnicas de classificação voltada ao objeto para mapear a variação temporal do uso e cobertura do solo a fim de subsidiar o entendimento do colapso hídrico em curso no Lago Poopó. Esse lago é o segundo maior lago da Bolívia e uma importante fonte de água para a população, no entanto, vem passando por uma forte crise hídrica desde 2010 com períodos de seca completa.

Assim, inicialmente essa pesquisa avaliou o desempenho dos classificadores MaxVer (Máxima Verossimilhança) para a classificação supervisionada pixel a pixel e os algoritmos SVM (Support Vector Machines) e KNN (KNearest Neighbor) para classificação orientada a objeto em imagens do sensor Operational Land Imager (OLI) do satélite Landsat-8 e do sensor Thematic Mapper (TM) do satélite Landsat-5 nessa região. Em um segundo momento, foi realizado o mapeamento de uso e cobertura do solo da região da sub- bacia P6 do Lago Poopó na Bolívia nos últimos 30 anos para compreensão da dinâmica de ocupação na área.

\section{ÁREA DE ESTUDO}

A bacia hidrográfica do Lago Poopó, situada nos Andes boliviano, faz parte da bacia endorreica do Altiplano (Figura 1a) e tem uma área de aproximadamente $15.000 \mathrm{~km}^{2}$.

O lago é pouco profundo, apresentando uma média de $1,08 \mathrm{~m}$, sendo a maior entrada de água feita pelo rio Desaguadeiro (60\% do total) e a saída se dá por um exutório na porção sul do Lago Poopó na direção dos salares de Coipasa e de Uyuni pelo rio Lakajawira (Figura 1b). Entretanto, há anos não é registrada a saída de água do lago para os salares (Satge, 2017). O clima da região é semiárido com menor precipitação (200 a $750 \mathrm{~mm} / \mathrm{ano}$ ) que evapotranspiração (1.500 mm/ano), essa ocorrendo majoritariamente nos corpos d'água superficiais (Satgé, 2017; Pillco et al., 2007; Garreaud et al., 2003). Desse modo, pequenas variações no balanço hídrico podem impactar fortemente a região.

A área de estudo, em que foram testados os classificadores e gerado o mapeamento de uso e cobertura do solo, é uma sub- bacia do Lago Poopó, denominada P6 (Figura 2b), que apresenta 355,6 $\mathrm{km}^{2}$ e está situada na porção central da bacia hidrográfica do lago (Figura 2a). A área apresenta representatividade no contexto da região por possuir áreas naturais (água, sedimento e vegetação nativa) e agricultáveis.

\section{MATERIAIS E MÉTODOS}

A cena que recobre a sub-bacia P6 é a 233/73 e, para o estudo de comparação dos classificadores, foram escolhidas as imagens do sensor TM de 06 de julho de 2003 e do sensor OLI do satélite Landsat-8 de 04 de Maio de 2015. A segunda etapa do trabalho, a análise temporal do uso e ocupação do solo, utilizou imagens adquiridas nos anos de 1985, 1990, 1995, 2000, 2005 e 2010 do satélite Landsat-5 e dos anos de 2015 e 2017 do satélite Landsat-8 (Tabela 1).
Todas as imagens foram adquiridas gratuitamente pelo site Earth Explorer do Serviço Geológico dos Estados Unidos (USGS).

As classes consideradas no mapeamento da cobertura do solo abrangeram vegetação nativa, a margem do lago não ocupada por água (área exposta), água, agricultura e sedimento de rejeito, a escolha das classes foi devida a grande representatividade dessas na área da sub-bacia, assim como em toda a bacia do Lago Poopó. 

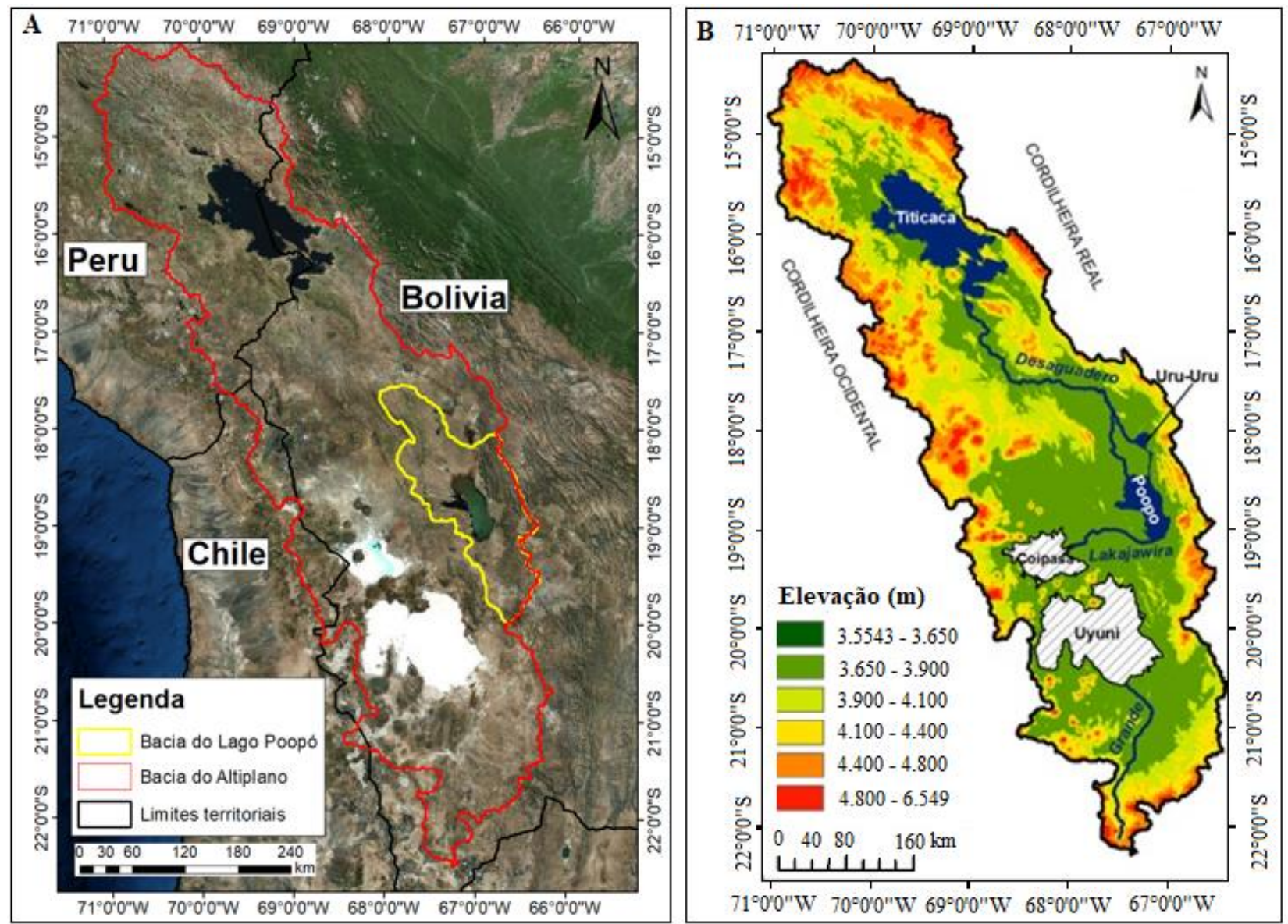

Figura 1 - (a) Mapa de localização da Bacia do Altiplano (em vermelho) e da Bacia do Lago Poopó (em amarelo) (b) Mapa de elevação indicando a localização dos principais lagos (Titicaca e Poopó), rios (Desaguardeiro, Lakajawi) do altiplano (modificado de Satgé, 2017).

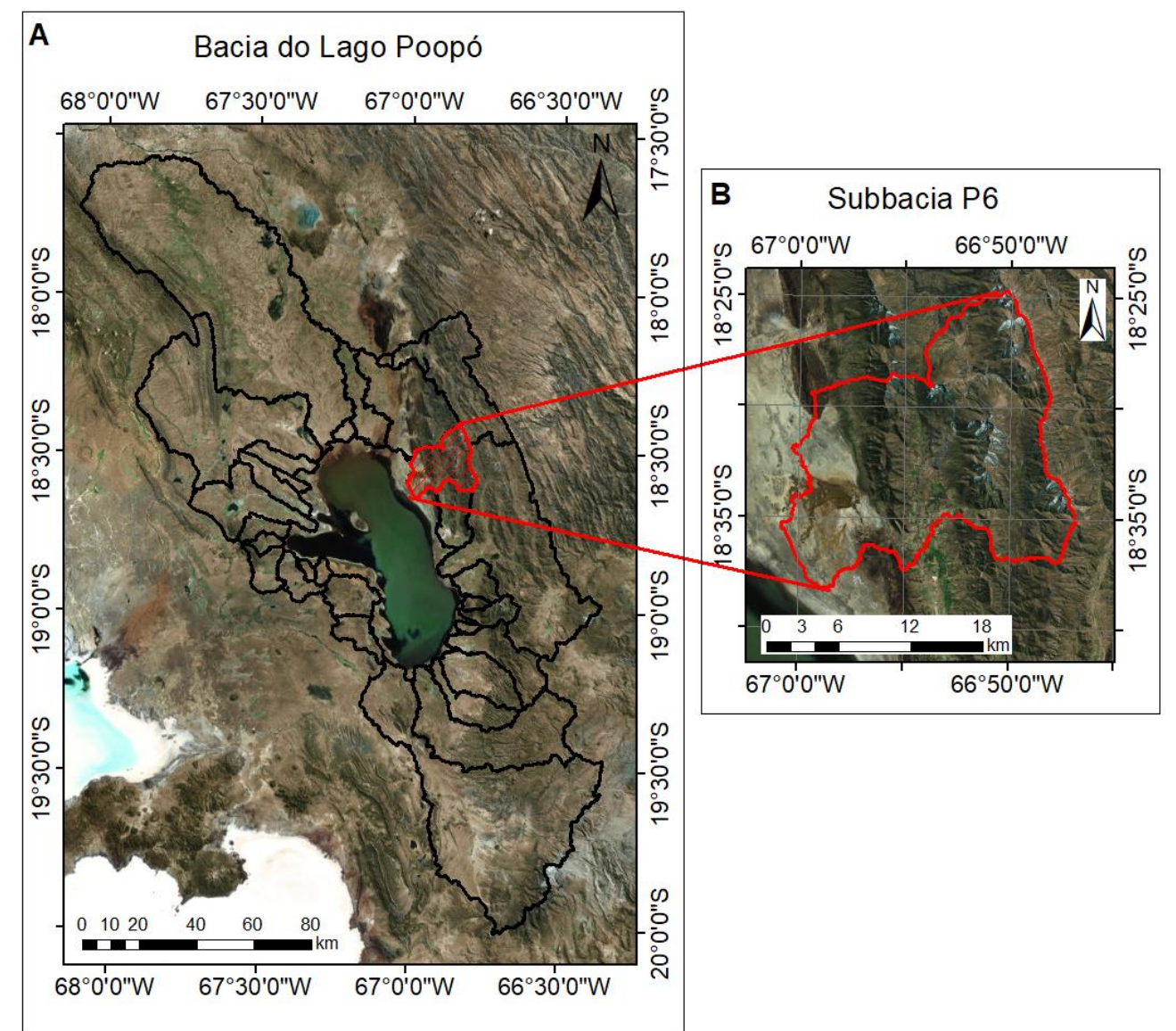

Figura 2 - Mapa de localização (a) da bacia do Lago Poopó (b) da subbacia P6 (destacado em verde). 
Tabela 1 - Datas das imagens utilizadas para o mapeamento temporal do Uso e Cobertura do Solo na subbacia P6.

\begin{tabular}{c|c}
\hline OLI/Landsat 8 & TM/Landsat 5 \\
\hline $16 / 05 / 2017$ & $06 / 05 / 2010$ \\
\hline $04 / 05 / 2015$ & $08 / 05 / 2005$ \\
\hline & $10 / 05 / 2000$ \\
\hline & $27 / 04 / 1995$ \\
\hline & $29 / 04 / 1990$ \\
\hline & $20 / 05 / 1985$ \\
\hline
\end{tabular}

A avaliação da variação temporal do uso e cobertura do solo nessa sub-bacia foi realizada em duas etapas (Figura 3). A primeira etapa consistiu na avaliação de classificadores e limiares para a definição de uma metodologia com alto grau de assertividade.

$\mathrm{Na}$ segunda etapa foi realizada, a partir do resultado da primeira fase, a classificação das imagens.

O pré-processamento envolveu rotinas de correções geométricas, radiométricas e atmos- féricas. A correção atmosférica foi realizada por meio do fast line of sight atmospheric analysis of spectral hypercubes (FLAASH) que utiliza o código de transferência radiativa moderate resolution transmittance (ModTran) para a conversão das radiâncias em reflectâncias na superfície terrestre (AdlerGolden et al., 1999; Berk et al., 2002; Fernandes et al., 2012).

Foram testados os classificadores pixel-apixel MaxVer e a ferramenta Feature Extraction (FX - ENVI). O MaxVer considera a ponderação das distâncias entre as médias dos valores dos pixels das classes, utilizando parâmetros estatísticos (INPE, 2008). As amostras para treinamento (ROI) do classificador MaxVer foram definidas com base em padrões característicos de cada classe (vegetação nativa, área exposta, água, agricultura e sedimento de rejeito).
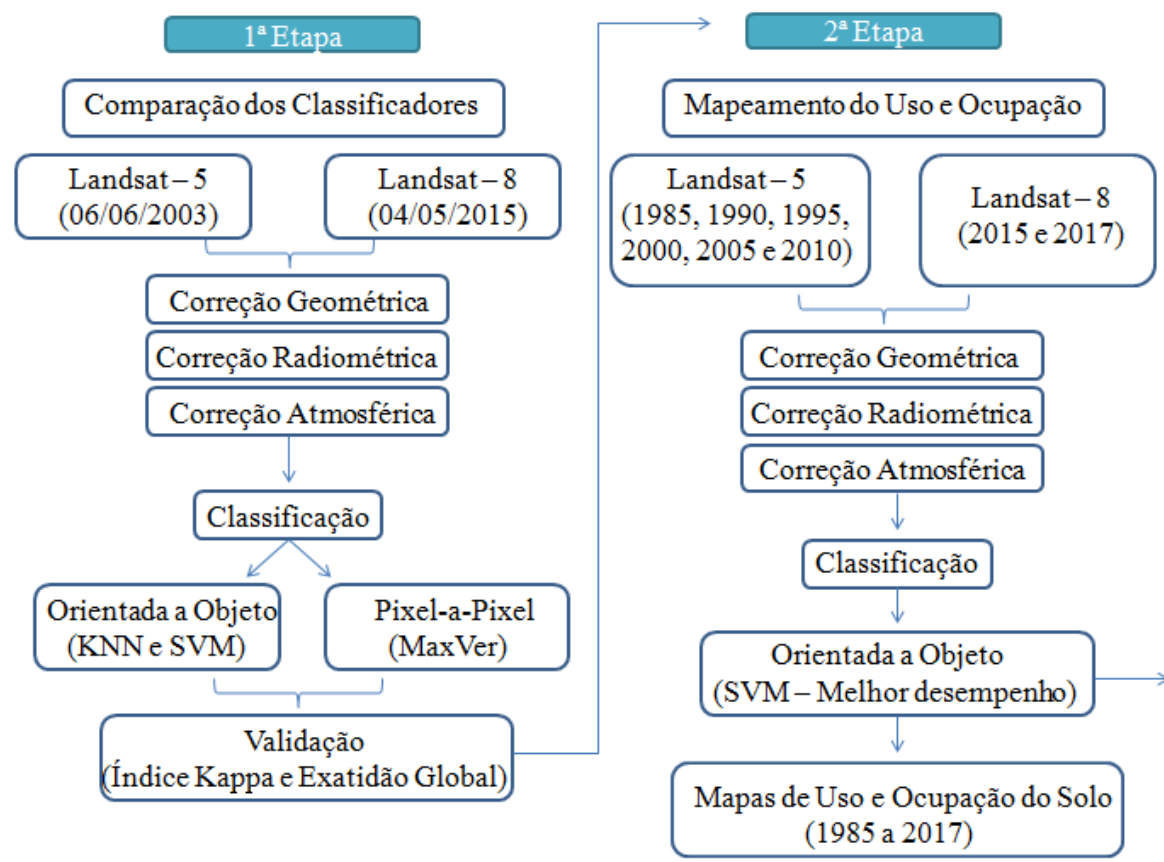

Figura 3 - Fluxograma de trabalho.

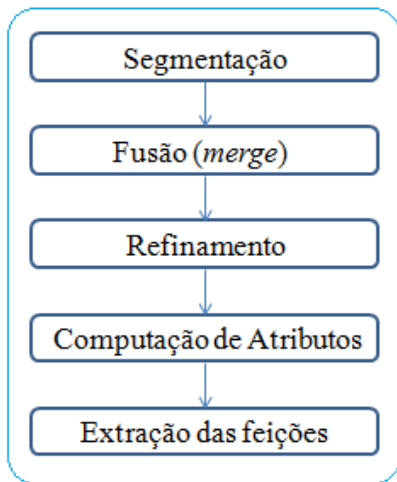

Para os classificadores por segmentação voltada a objeto, a ferramenta Feature Extraction possibilita extrair informações de objetos específicos de interesse com base nas características espaciais, texturais e espectrais das imagens pancromáticas ou multiespectrais. São ao todo cinco distintas etapas: (i) segmentação, (ii) fusão (merge), (iii) refinamento, (iv) computação de atributos e (v) extração das feições por regras ou por exemplos (ENVI, 2008).

A segmentação é o processo de particionamento de uma imagem em objetos pelo agrupamento de pixels vizinhos, com valores comuns (Harris Geospatial Solutions, 2018) e quanto mais efetiva for a segmentação, melhor serão os resultados da classificação (Campos et al., 2013).

Nas imagens da série LANDSAT processadas nesse trabalho foram utilizadas o algoritmo Edge que, segundo Harris Geospatial Solutions (2018), melhor segmenta objetos que possuem arestas. Os limiares de cada ano encontram-se listados na tabela 2.

A etapa de fusão teve como princípio agrupar os segmentos gerados por similaridade e adjacência. O algoritmo "Full Lambda 
Schedule" combina pequenos segmentos em áreas maiores e texturizadas. Esse algoritmo foi utilizado na pesquisa com limiar de 10 para todas as imagens nessa etapa. Tanto para a segmentação quanto para a fusão os limiares foram escolhidos após uma detalhada avaliação visual das imagens que recobrem a área. Nesse trabalho os atributos espaciais, texturais e espectrais, foram explorados e utilizados em sua totalidade ou apenas parcialmente (maior relevância) (Tabela 3 ).

Tabela 2 - Limiares utilizados na segmentação das imagens para cada ano.

\begin{tabular}{c|c|c|c|c|c|c|c}
\hline \multicolumn{7}{c}{ Segment Setting } \\
\hline $\mathbf{2 0 1 7}$ & $\mathbf{2 0 1 5}$ & $\mathbf{2 0 1 0}$ & $\mathbf{2 0 0 5}$ & $\mathbf{2 0 0 0}$ & $\mathbf{1 9 9 5}$ & $\mathbf{1 9 9 0}$ & $\mathbf{1 9 8 5}$ \\
\hline 40 & 40 & 30 & 40 & 25 & 40 & 40 & 40 \\
\hline
\end{tabular}

Tabela 3 - Tabela dos atributos que o ENVI FX utilizada para a classificação dos segmentos (*os atributos espectrais e texturais são aplicadas para todas as bandas das imagens).

\begin{tabular}{|c|c|c|}
\hline Espacial & Espectral* & Textural* \\
\hline Área & Mínima (Spectral Min) & Escala (Texture Range) \\
\hline Comprimento & Máxima (Spectral Max) & Média (Texture Mean) \\
\hline Compacidade & Média (Spectral Mean) & Variância (Texture variance) \\
\hline Convexidade & Std (Spectral Std) & Entropia (Texture Entropy) \\
\hline \multicolumn{3}{|l|}{ Solidez } \\
\hline \multicolumn{3}{|l|}{ Esfericidade } \\
\hline \multicolumn{3}{|l|}{ Fator } \\
\hline \multicolumn{3}{|l|}{ Elongação } \\
\hline \multicolumn{3}{|l|}{ Forma Retangular } \\
\hline \multicolumn{3}{|l|}{ Direção Principal } \\
\hline \multicolumn{3}{|l|}{ Maior Comprimento } \\
\hline \multicolumn{3}{|l|}{ Menor Comprimento } \\
\hline \multicolumn{3}{|l|}{ Número de buracos } \\
\hline Área de buracos/Área solida & & \\
\hline
\end{tabular}

A extração dos objetos foi realizada utilizando "exemplos", assim a classificação foi definida a partir de um processo que associa dados desconhecidos, a uma ou mais feições conhecidas (ENVI, 2008; Nunes \& Roig, 2014). Os dados de treinamento consistiram em objetos (segmentos) selecionados como elementos amostrais representativos das classes de vegetação natural, sedimento de rejeito, água, área exposta e agricultura. Para cada classe foi coletado uma quantidade representativa de exemplos para servirem de base para o algoritmo classificar automaticamente o restante da imagem.

Os algoritmos testados para a classificação de toda a imagem foram o KNN (K-Nearest Neighbor) e SVM (Support Vector Machine). O KNN é um classificador no qual o aprendizado é baseado na analogia. O conjunto de treinamento é formado por vetores n-dimensionais e cada elemento deste conjunto representa um ponto no espaço n-dimensional (Gnecco et al., 2005). O
SVM é embasado pela teoria de aprendizado estatístico, essa teoria estabelece uma série de princípios que devem ser seguidos na obtenção de classificadores com boa generalização, definidos como a sua capacidade de prever corretamente a classe de novos dados do mesmo domínio em que o aprendizado ocorreu (Lorena e Carvalho, 2007).

As avaliações da metodologia de maior assertividade nas imagens de 2003 (Landsat 5/ TM) e 2015 (Landsat 8/ OLI) utilizando classificação pixel-a-pixel e por segmentação foram realizadas com a análise da qualidade da classificação de acordo com o índice Kappa (Landis e Kock, 1977) e a Exatidão Global (Jensen, 1996), a partir das matrizes de confusão. A escolha das datas das imagens utilizadas na análise comparativa de classificadores teve como premissa básica o período em que o Google Earth apresentava o melhor recobrimento na região. Para a validação foram gerados 200 pontos na área da sub-bacia e essas amostras 
foram geradas de forma aleatória com o apoio da ferramenta "Create Random Points" no software ArcMap 10.3 e validadas por imagens do Google Earth dos anos analisados.

Assim, após a avaliação estatística foi elaborada a metodologia e replicada nas imagens entre 1985 e 2017 resultando na classificação de uso e cobertura do solo dos últimos 30 anos. A segunda etapa da pesquisa resultou na geração de oito imagens classificadas que foram comparadas entre si para o entendimento da dinâmica espaço temporal da sub-bacia P6.

\section{RESULTADOS E DISCUSSÕES}

Desempenho dos Algoritmos de Classificação

A etapa de avaliação do desempenho dos algoritmos de classificação SVM, KNN e Maxver nas imagens TM e OLI (2003 e 2017, respectivamente) foi realizada utilizando os parâmetros apresentados na seção metodológica desse trabalho.

Em relação as classificações utilizando o
SVM e KNN foram realizados testes na parte de segmentação com a utilização de todos os atributos espaciais, texturais e espectrais e com a utilização somente dos atributos de maior relevância utilizando como apoio a ferramenta de autosseleção do programa; os atributos que foram selecionados para cada imagem encontram-se na tabela 4.

Tabela 4 - Tabela dos atributos que o Envi FX (ferramenta de autosseleção) selecionou para a classificação.

\begin{tabular}{|c|c|c|}
\hline \multicolumn{3}{|c|}{ TM/L5 - 2003} \\
\hline \multicolumn{2}{|c|}{ Espectral } & Textural \\
\hline \multicolumn{2}{|c|}{ Mínima (Spectral Min) } & Escala (Texture Range) \\
\hline \multicolumn{2}{|c|}{$\mathrm{B} 1, \mathrm{~B} 2, \mathrm{~B} 3, \mathrm{~B} 4, \mathrm{~B} 5, \mathrm{~B} 7$} & $\mathrm{~B} 5, \mathrm{~B} 7$ \\
\hline \multicolumn{2}{|c|}{ Máxima (Spectral Max) } & Média (Texture Mean) \\
\hline \multicolumn{2}{|c|}{$\mathrm{B} 2, \mathrm{~B} 3, \mathrm{~B} 3, \mathrm{~B} 5, \mathrm{~B} 7$} & $\mathrm{~B} 1, \mathrm{~B} 2, \mathrm{~B} 3, \mathrm{~B} 4, \mathrm{~B} 5, \mathrm{~B} 7$ \\
\hline \multicolumn{2}{|c|}{ Média (Spectral Mean) } & Variância (Texture variance) \\
\hline \multicolumn{2}{|c|}{$\mathrm{B} 1, \mathrm{~B} 2, \mathrm{~B} 3, \mathrm{~B} 4, \mathrm{~B} 5, \mathrm{~B} 7$} & $\mathrm{~B} 4, \mathrm{~B} 5, \mathrm{~B} 7$ \\
\hline \multicolumn{2}{|c|}{ Std (Spectral Std) } & Entropia (Texture Entropy) \\
\hline $\mathrm{B} 1, \mathrm{~B} 5, \mathrm{~B} 7$ & \multicolumn{2}{|c|}{ B1,B2,B3,B4,B5,B7 } \\
\hline \multicolumn{3}{|c|}{ OLI/L8 - 2015} \\
\hline Espacial & Espectral & Textural \\
\hline Maior Comprimento & Mínima (Spectral Min) & Escala (Texture Range) \\
\hline Comprimento & $\mathrm{B} 1, \mathrm{~B} 2, \mathrm{~B} 3, \mathrm{~B} 4, \mathrm{~B} 5$ & B1,B2,B3,B4,B5,B6,B7 \\
\hline \multirow[t]{6}{*}{ Fator (From Factor) } & Máxima (Spectral Max) & Média (Texture Mean) \\
\hline & B2,B3,B3,B5,B7 & $\mathrm{B} 1, \mathrm{~B} 2, \mathrm{~B} 3, \mathrm{~B} 4, \mathrm{~B} 5, \mathrm{~B} 6, \mathrm{~B} 7$ \\
\hline & Média (Spectral Mean) & Variância (Texture variance) \\
\hline & B1,B2,B3,B4,B5,B6,B7 & $\mathrm{B} 5, \mathrm{~B} 6$ \\
\hline & Std (Spectral Std) & Entropia (Texture Entropy) \\
\hline & $\mathrm{B} 5, \mathrm{~B} 6, \mathrm{~B} 7$ & $\mathrm{~B} 5, \mathrm{~B} 6$ \\
\hline
\end{tabular}

O resultado do índice kappa e da exatidão global das classificações (Tabela 5), gerados a partir das matrizes de confusão, mostra que o classificador digital que apresentou melhor acurácia foi o SVM, utilizando somente os atributos de maior relevância para a classificação das imagens. De acordo com Landis e Kock (1977), os valores do índice Kappa, indicam que a qualidade de todas as classificações geradas é de muito boa a excelente. Mas segundo Anderson et al., 2001, a exatidão global das classificações geradas pelo classificador MaxVer não apresentou valor aceitável (mínimo admissível $\geq 0,85$ ).
Ao se comparar os valores do Índice Kappa e da Exatidão Global das classificações geradas nas imagens do sensor OLI/Lansat-8 e TM/Landsat-5, é possível observar que os resultados apresentam variações sutis para todos os algoritmos utilizados (Tabela 5), demonstrando que os classificadores se comportaram de forma semelhante para ambas as imagens. Um dos principais problemas identificados foi em função da área de estudo ser muito acidentada, fazendo com que a imagem apresentasse uma grande quantidade de sombras, assim na classificação toda a região sombreada 
foi caracterizada como vegetação nativa.

A análise das matrizes de confusão para cada classificação indica que as classes melhor definidas foram áreas expostas, bacias de sedimentação de rejeito e água. As maiores inconsistências foram observadas para as classes destinadas à agricultura e a vegetação nativa, provavelmente associadas ao comportamento espectral semelhante dessas classes. A associação de atributo de "forma" na classificação reduz a confusão, mas ainda torna a classificação dessa classe muito duvidosa.

Tabela 5 - Resultados das validações das classificações. KNN11 e SVM11 utilizando todos os atributos e KNN² e SVM² utilizando os atributos selecionados.

\begin{tabular}{|c|c|c|c|c|c|}
\hline \multicolumn{6}{|c|}{ Índice Kappa (\%) } \\
\hline & $\mathrm{KNN}^{1}$ & $\mathrm{KNN}^{2}$ & SVM1 & SVM $^{2}$ & MAXVER \\
\hline $\begin{array}{l}\mathbf{L} \\
5\end{array}$ & 78,76 & 79,65 & 79,32 & 83,7 & 67,94 \\
\hline $\begin{array}{l}\mathbf{L} \\
8\end{array}$ & 79,15 & 82,27 & 81,64 & 82,28 & 69,82 \\
\hline \multicolumn{6}{|c|}{ Exatidão Global (\%) } \\
\hline & KNN $^{1}$ & $\mathrm{KNN}^{2}$ & SVM1 & SVM $^{2}$ & MAXVER \\
\hline $\begin{array}{l}\mathbf{L} \\
5\end{array}$ & 87,5 & 89,5 & 89,5 & 92 & 83,5 \\
\hline $\begin{array}{l}\mathbf{L} \\
8\end{array}$ & 88,5 & 90,5 & 90 & 92 & 83 \\
\hline
\end{tabular}

(1) resultados gerados utilizando todos os atributos.

(2) resultados gerados utilizando apenas os atributos mais relevantes de acordo com a seleção automática do ENVI-FX.

O algoritmo de melhor qualidade na classificação e que apresentou a menor confusão entre as classes foi a que utilizou o algoritmo SVM com a seleção de atributos, o que coincide com os resultados do índice kappa e da exatidão global. O alto desempenho do SVM, com menor erro de classificação das classes se deve à robustez e capacidade de formar planos $n$ - dimensionais deste método (Garofalo et al., 2015).

\section{Mapeamento do Uso e Cobertura do Solo}

Após o teste dos algoritmos e a análise estatística da validação do índice Kappa e da Exatidão Global, verificou-se que o melhor algoritmo é o SVM utilizando a seleção automática de atributos do software, portanto foi esse algoritmo, com o apoio da ferramenta de autosseleção de atributos, que foi utilizado para a classificação de uso e ocupação do solo nos anos de 1985, 1990, 1995, 2000, 2005, 2010, 2015 e 2017.

Os resultados das classificações (Figuras 4 e 5) indicam que a classe vegetação nativa é a predominante na sub-bacia P6 do Lago Poopó, incluem-se nessa classe vegetação de montanha e estepe (vegetação típica dos Andes, formada basicamente por gramíneas), atualmente está classe representa $65,91 \%\left(234,45 \mathrm{~km}^{2}\right)$ do total da área da sub-bacia, mas em 1985 representava $73,66 \%\left(262,02 \mathrm{~km}^{2}\right)$. Essa cobertura natural foi substituída principalmente por áreas de agricultura. Em 2017 a agricultura representou $13,52 \%\left(48,10 \mathrm{~km}^{2}\right)$ da sub-bacia, valor este muito superior, quando se comparada a $1985 \mathrm{em}$ que essa classe correspondia apenas 5\% $\left(15,44 \mathrm{~km}^{2}\right)$ do total da área. As áreas de vegetação nativa são importantes, pois conferem proteção ao solo, diminuindo a velocidade de escoamento superficial e favorecendo a infiltração de água no solo.

A classe água também apresentou uma grande variação temporal, em 2017 representava apenas $0,06 \%\left(0,216 \mathrm{~km}^{2}\right)$ do total da área da sub-bacia, entretanto em 1985 representava $6 \%\left(14,99 \mathrm{~km}^{2}\right)$ desse total; simultaneamente a essa perda de massa d'água, houve o aumento da classe de área exposta que passou de $14,81 \%\left(52,70 \mathrm{~km}^{2}\right) \mathrm{em}$ 1985 , para $18,67 \%\left(66,40 \mathrm{~km}^{2}\right)$ em 2017 . A área de sedimentação de rejeito na região de estudo (incluída na classe de área exposta) é oriunda principalmente das atividades de mineração, essa classe foi a que apresentou a menor variação ao longo dos anos.

Em toda a bacia do altiplano o "boom" da quinoa começou no início dos anos 2000 (Satgé, 2017). Entretanto ao analisar a dinâmica do uso da terra na sub-bacia P6, o aumento das áreas agrícolas começou após 2010 e a perda de área úmida na região de estudo vem ocorrendo desde 1995 (Figura 6a), 15 anos antes do aumento das atividades agrícolas e diminuição das áreas de vegetação nativa na sub-bacia (Figura 6b). Desta 
forma, diversos fatores podem estar contribuindo com essa redução acelerada dos corpos de água, como por exemplo, as variações climáticas locais e as atividades antrópicas como a mineração, a extração de água subterrânea e superficial de forma desordenada e a agricultura em outras regiões da bacia do lago Poopó que interfere no ciclo hidrológico de forma regional.

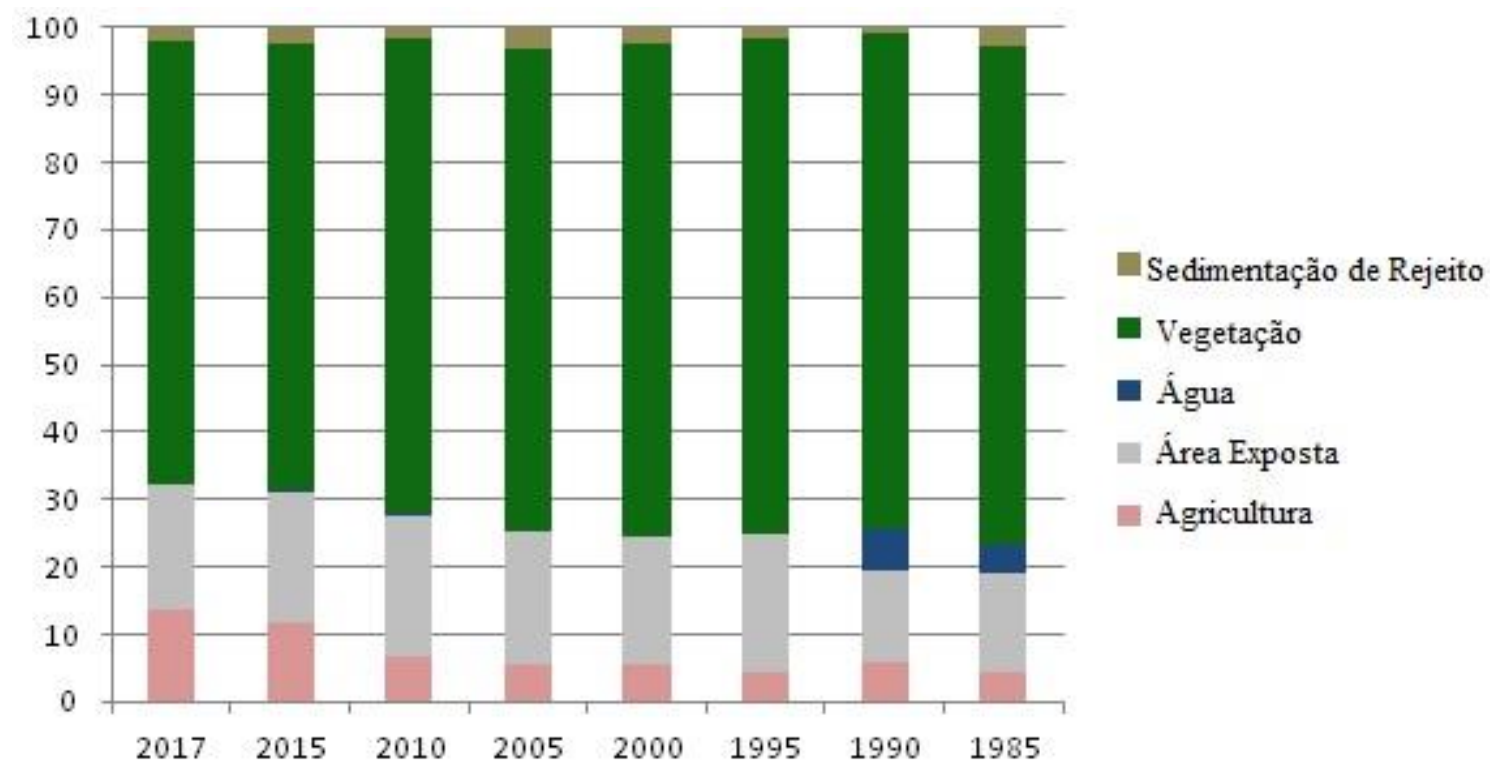

Figura 4 - Gráfico indicando a representatividade de cada classe (em \%) nos mapas de uso e cobertura do solo.

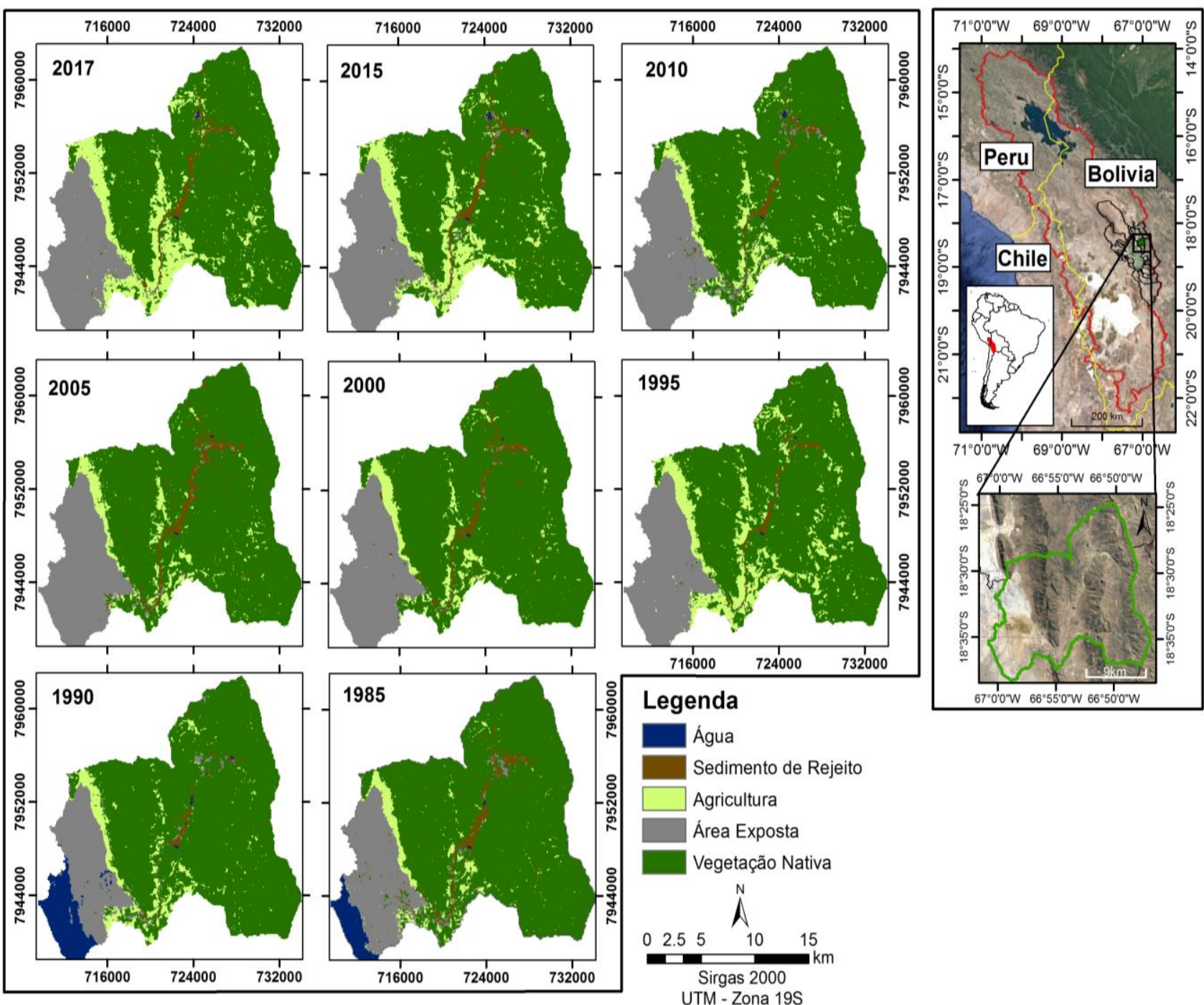

Figura 5 - Mapas de Uso e Ocupação do Solo na sub-bacia do Lago Poópo de 1985 a 2017. 

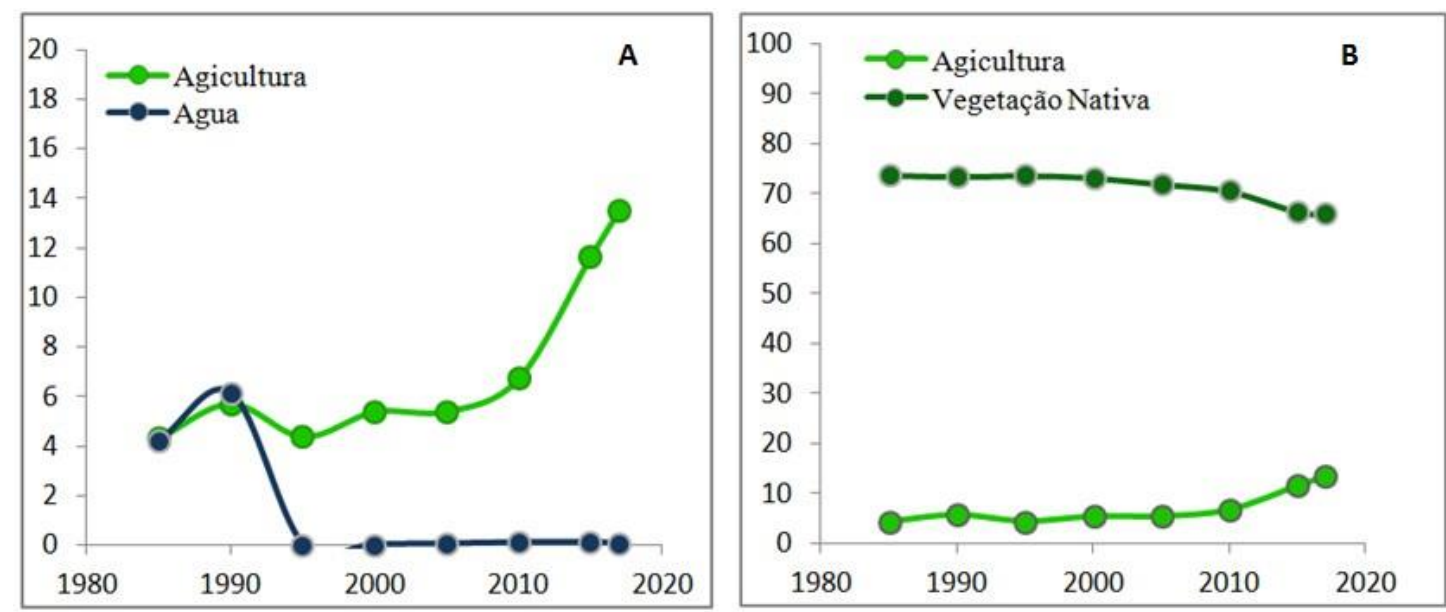

Figura 6 - Gráficos de dispersão (\%) (a) comparando a classe agricultura com a água (c) comparando a classe vegetação nativa com agricultura.

\section{CONCLUSÕES}

1. As classificações voltadas ao objeto utilizando os algoritmos SVM e KNN, são mais eficientes que a classificação supervisionada pixe-a-pixel MaxVer; sendo o classificador SVM com atributos de maior relevância com desempenho superior ao $\mathrm{KNN}$;

2. O ENVI FX se apresentou uma ferramenta automática de fácil e rápido manuseio e com resultados de qualidade.

3. Os valores do índice Kappa e da Exatidão Global, indicam que a qualidade das classificações geradas pelos classificadores SVM e KNN é de muito boa a excelente. Desta forma, classificou com exatidão as cinco classes previamente definidas para a sub-bacia P6 do Lago Poopó, e viabilizou a obtenção da caracterização de uso e cobertura do solo;

4. A região de estudo em 2017 apresentou aproximadamente $65,9 \%$ de sua área coberta de vegetação nativa, $13,5 \%$ por agricultura, $0,06 \%$ de corpos d'água, $18,74 \%$ de área exposta e 1,8\% da área representa sedimentação de rejeito;
5. A redução da área da classe "água” vem ocorrendo desde 1995, 15 anos antes do aumento significativo das atividades agrícolas e diminuição das áreas de vegetação nativa que foi potencializada após 2010; No entanto, considerando a bacia do altiplano, e especialmente a região do lago Titicaca, a diminuição das áreas de vegetação nativa aliado ao aumento das atividades agrícolas e da irrigação foi registrado desde os anos 2000 (Satgé, 2017) o que sugere que é preciso considerar uma escala mais regional para entender as causas da seca do lago Poopó.

Diversos fatores podem estar contribuindo com a aceleração da perda de água no lago, como, por exemplo, as variações climáticas regionais, a extração de água subterrânea e superficial de forma desordenada, a agricultura em outras regiões da bacia do altiplano e as atividades antrópicas como a mineração, que interfere no ciclo hidrológico de forma regional.

\section{AGRADECIMENTOS}

Agradecemos a Universidade de Brasília e o Joint International Laboratory (LMI) pelo apoio institucional. E a todos os professores do curso de Especialização em Geoprocessamento Ambiental (UnB).

\section{REFERÊNCIAS}

ADLER-GOLDEN. S.M.; MATTHEW. M.W.; BERNSTEIN. L.S.; LEVINE. R.Y.; BERK. A.; RICHTSMEIER. S.C.; ACHARYA. P.K.; ANDERSON. G.P.; FELDE. G.W.; GARDNER. J.; HOKE. M.; JEONG. L.S.; PUKALL. B.; MELLO. J.; RATKOWSKI. A.; BURKE. H.-H. Atmospheric correction for short-wave spectral imagery based on MODTRAN4. Proceedings of the Society of Photo-Optical Instrumentation Engineers. v.3753. p.61-69. 1999.

ANDERSON. J.F.; HARDY. E.E.; ROACH. J.T.; WITMER R.E. $A$ land use and land cover classification system for use with remote sensor data. U.S. Geological Survey Professional Paper
964. Washigton: U.S. Geologic Survey.2001. 41p (paper 964). BERK. A.; ADLER-GOLDEN. S.M.; RATKOWSKI. A.; FELDE. G.W.; ANDERSON. G.P.; HOKE. M.L.; COOLEY. T.; CHETWYND. J.H.; GARDNER. J.A.; MATTHEW. M.W.; BERNSTEIN. L.S; ACHARYA. P.K.; MILLER. D.; LEWIS. P. Exploiting MODTRAN radiation transport for atmospheric correction: the FLAASH algorithm. In: INTERNATIONAL CONFERENCE ON INFORMATION FUSION. 5.. 2002. Annapolis. Proceedings. Annapolis: ISIF. 2002. v.2. p.798-803. BLAIKIE, P.M. and BROOKFIELD, H. 2015: Land degradation and society. London: Methuen. 196p. 
BLASCHKE, T.; KUX, H. J. H., 2007. Sensoriamento remoto e SIG avançados - novos sistemas sensores: métodos inovadores. Oficina de Textos, São Paulo, $2^{\mathrm{a}}$ ed. 303 p.

BOMMARCO R., KLEIJN D., POTTS S.G. (2013) Ecological intensification: harnessing ecosystem services for food security. Trends Ecol Evol 28: 230-238.

CAMARGO. F.F.; ALMEIDA.C.M.; COSTA. G.A.O.P.; FEITOSA. R.Q.; OLIVEIRA. D.A.B.; HEIPKE. C.; FERREIRA. R.S. An open source object-based framework to extract landform classes. Expert Systems with Applications. v. 39. n. 1. p. 541-554. 2012.

CAMPOS. A.R.. FONSECA. D.R.. SAMPAIO. D.M.. FARIA. S.D.. ELMIRO. M.A.T. Classificação orientada a objeto para mapeamento do uso e cobertura do solo do município de Rio Acima - MG: por crescimento de regiões e por extração de objetos. Anais XVI Simpósio Brasileiro de Sensoriamento Remoto - SBSR. Foz do Iguaçu. PR. Brasil. 13 a 18 de abril de 2013. INPE

DONATIO. N. M. M.; GALBIATTI. J. A.; DE PAULA. R. C. Qualidade da água de nascentes com diferentes usos do solo na bacia hidrográfica do córrego Rico. São Paulo. Brasil. Engenharia Agrícola. v.25. n.1. p.115-125. 2005.

ENVIRONMENTAL SYSTEMS RESEARCH INSTITUTE (ESRI). ArcGis Professional Gis for desktop, versão 10.3, 2018.

ENVI. ENVI FEATURE EXTRACTION MODULE: User's guide. [S.L.]: ITT visual information solution. 2008.

FERNANDES, R.R.; NUNES, G.M.; SILVA. T.S.F. "Classificação orientada a objetos aplicada na caracterização da cobertura da terra no Araguaia". Pesq. Agropec. Bras. Brasília. v.47. n.9. p.1251-1260. set. 2012.

GARREAUD. RENÉ. MATHIAS VUILLE. and Amy C. Clement. 2003. "The Climate of the Altiplano: Observed Current Conditions and Mechanisms of Past Changes." Palaeogeography. Palaeoclimatology. Palaeoecology 194 (13): 5-22. doi:10.1016/S0031-0182(03)00269-4.

GAROFALO. D.F.T.. MESSIAS. C.G.. LIESENBERG. V. BOLFE. E. L.. FERREIRA. M. C. "Análise comparativa de classificadores digitais em imagens do Landsat-8 aplicados ao mapeamento temático.” Pesq. Agropec. Bras.. Brasília. v.50. n.7. p.593-604. jul. 2015 DOI: 10.1590/S0100-204X2015 000700009.

GNECCO. B.B. Um Sistema de Visualização Imersivo e Interativo de Apoio ao Ensino de Classificação de Imagens. disponível em http://www.di.ufpb.br/liliane/ publicacoes/wrv 2001-cave-final.pdf. acesso em 25/05/2005.

HARRIS GEOSPARIAL SOLUTIONS. EXTRACT FEATURE. Disponível em <http://www.harrisgeospatial.com/docs/Extract Features.html>. Acesso em Fevereiro de 2018.

INSTITUTO NACIONAL DE PESQUISAS ESPACIAIS (INPE) - Manuais: tutorial de geoprocessamento SPRING. 2008.

JENSEN. J.R.. 1996. Introductory digital image processing: a remote sensing perspective. Prentice-Hall. Upper Saddle River. NJ. 318 p

LANDIS. J.R. \& KOCK. G. G. The measurement of observer agreement for categorical data. Biometrics. 33: 159-75. 1977.
LORENA, A.C., CARVALHO, A.C.P.L.F. Uma Introdução às Support Vector Machines. Rev. Informática Teórica e Aplicada: Volume XIV, Número 2. 25p. 2007.

LUZ. N.B. DA; ANTUNES. A.F.B.; TAVARES JÚNIOR. J.B. Segmentação multirresolução e classificação orientada a objetos aplicados a imagens Spot-5 para o mapeamento do uso da terra. Floresta. v.40. p.429-446. 2010.

MENESES. P. R.; ALMEIDA. T. D. (Organizadores). Introdução ao Processamento de Imagens de Sensoriamento Remoto. Universidade de Brasília. Brasília. 2012. 266 p.

NUNES. F.N. E ROIG.H. L. "Análise e Mapeamento do Uso e Ocupação do Solo da Bacia do Alto do Descoberto. DF/GO por meio de classificação automática baseada em regras e lógica nebulosa." Revista Árvore. Viçosa-MG. v.39. n.1. p.25-36. 2014. Dói: 10.1590/0100-67622015000100003.

PILLCO R. CINTIA B.U. AND LARS B. 2007. "Precipitation Variability and Regionalization over the Southern Altiplano. Bolivia.” International Journal of Climatology. 149-64.

RESTREPO, JOSÉ J. BARRIOS ; FLOHR, LETÍCIA ; MELEGARI, SILVIA P. ; DA COSTA, CRISTINA H. ; FUZINATTO, CRISTIANE F. ; DE CASTILHOS JR., ARMANDO B. ; MATIAS, WILLIAM G. . Correlation between Acute Toxicity for Daphnia magna, Aliivibrio fischeri and Physicochemical Variables of the Leachate Produced in Landfill Simulator Reactors. Environmental Technology, v. 1, p. 1-28, 2017.

SATGÉ. F.C. Recursos Hídricos Do Altiplano Andino: Contribuição Do Sensoriamento Remoto. Universidade de Brasília (Tese de Doutorado). 2017. 213 p.

SANO. E.E.; ROSA. R.; BRITO. J.L.S.; FERREIRA. L.G; BEZERRA. H.S. Mapeamento da cobertura vegetal natural e antrópica do bioma Cerrado por meio de imagens Landsat ETM+. In: SIMPÓSIO BRASILEIRO DE SENSORIAMENTO REMOTO. 14.. 2009. Natal. Anais... Natal: INPE. 2009. p.1199-1206.

SMALL, I., VAN DER MEER, J., UPSHUR, R.E.G., 2001. Acting on an environmental health disaster: The case of the Aral Sea. Environ. Health Perspect. 109, 547-549. doi:10.1289/ehp.01109547.

TURNER, B.L.; LAMBIN, E.F. \& REENBERG, A. (2007). The Emergence of Land Change Science for Global Environmental Change and Sustainability. Proc. Nat. Acad. Scien. USA (PNAS). 105. 20690-20695. 